\title{
An X-ray Diffraction Method for Quantitative Determination of Retained Austenite in the Production Line of Metastable
} Austenitic Stainless Steel*

\author{
By Hajime KITAGAWA** and Taiji SOHMURA $A^{* * *}$
}

\begin{abstract}
Synopsis
An X-ray diffraction method has been developed for quantitative determination of retained austenite in the production line of cold-rolled type SUS 301 metastable austenitic stainless steel sheets. A new unit of diffractometer designed for application in the mill is equipped with an $X$-ray source of $\mathrm{Cr} \mathrm{K} \alpha$ and three separate scintillation detectors and is provided with mechanisms of lateral movement along the transverse direction of strip and rotation around an axis vertical to the strip surface. Intensities of $\{211\}$ martensite reflection, $\{110\}$ austenite reflection, and background are measured separately by the three detectors.

Measurements were carried out by scanning of a coil, cold rolled in a reversing mill, during uncoiling operation in a slitting line. Diffraction intensities of austenite phase were weaker in the top and tail ends than in the middle part of the coil. Variation of the diffraction intensities throughout the whole coil length is well correlated with a conceptional distribution of retained austenite which can be deduced from the nickel equivalent and rolling temperature of the tested material. This findings suggest applicability of the present method in the production line, even though the effect of textures is not corrected.

The new $X$-ray diffractometer unit is proved to be successfully employed in the production mill as a practical means for prediction of inhomogeneity of material properties in the stainless steel strip for use of springs by monitoring the relative amount of austenite and martensite.
\end{abstract}

\section{Introduction}

Mechanical properties of steels with two or more phases generally depend on the relative amounts of phases in the steel.

Relative amount of ferrite and martensite determines the yield and tensile strength of a high strength "dual-phase" steel sheet. Hardness and bending fatigue strength of a stainless steel for spring also depend on the relative amount of martensite and austenite.

This paper describes an X-ray diffraction method for quantitative analysis of volume fractions of phases in a production line of metastable austenitic stainless steel. The steel is specified as SUS 301 by JIS (Japanese Industrial Standard), identifiable with AISI 301.

The steel is processed by hot rolling and solution heat treatment, and finished by cold rolling to impart high strength required for the use of spring.

The cold rolling induces the martensite transformation of metastable austenite. The volume fraction of strain-induced martensite, which determines the material performance of the rolled product, is influenced by the chemical composition, the reduction, and temperature of rolling. As the rolling operation is not controlled to maintain a constant temperature in the ordinary cold rolling process, the rolling tem- perature may vary from room temperature to more than $150^{\circ} \mathrm{C}$ in a coil.

Thus the relative volume fraction of phases can not be constant within a coil and also among coils of an identical chemical composition, rolled with the same reduction. Accordingly, there arises a need for quantitative determination of retained austenite in the production line of the steel, in order to guarantee the uniformity of mechanical properties.

\section{Principle of Measurement}

The volume fractions of phases in materials have usually been evaluated by optical microscopy, magnetic analysis, and X-ray diffractometry.

Among these methods, the X-ray diffraction method is one of the most efficient means, even though these are usually employed only in laboratories.

The determination of volume fraction by the X-ray diffraction is based on the fact that X-ray diffraction intensities are directly proportional to the volume of the phase considered.

The most severe shortcoming with this method is the problem of calibration due to preferred orientation, since all materials in reality always have a preferred orientation to some extent.

The problem of quantitative phase analysis in textured material is one of obtaining the integrated intensity averaged over all orientations of the specimen with respect to the X-ray beam. Methods of averaging involve randomizing the intensities by mathematical or mechanical means.

A number of methods of averaging the intensities have been proposed for the quantitative phase analysis of textured material. ${ }^{1,2)}$

The method of Gullberg and Lagneborg') reduces the effects of preferred orientation by mathematical averaging of the integrated intensities of reflections of each phase for one single orientation of an alloy specimen containing two phases, $\alpha$ and $\gamma$.

It is possible to obtain the correct average intensity, $I \alpha$ or $I \gamma$, for a specific diffraction plane by performing an appropriate averaging intensities belonging to a large number of diffraction planes of different $\{h k l\}$ when all intensities have been produced from single orientation of the specimen surface. Substituting these corrected intensities, the volume fractions of phases, $V \alpha$ and $V \gamma$, and derived from the following equations:

\footnotetext{
* Presented at the 102nd ISIJ Meeting, November 1981, S1166, at Kyoto-fu Chusho Kigyo Kaikan in Kyoto. Manuscript received May 4, 1982. (C) 1983 ISIJ

** Research Laboratories, Kawasaki Steel Corporation, Kawasaki-cho, Chiba 260.

*** Research \& Development Laboratory, Rigaku Industrial Corporation, Akaōji-cho, Takatsuki 569.
} 


$$
\begin{aligned}
& V \alpha / V \gamma=(I \alpha R \gamma / I \gamma R \alpha) \\
& V \alpha+V \gamma=1
\end{aligned}
$$

where, $R \alpha, R \gamma$ : factors determined by the crystal structure of the particular phases and indices of the reflection.

The method of Miller ${ }^{2)}$ mechanically randomizes the intensities by using a specimen holder which simultaneously tilts and rotates a specimen in the path of the X-ray beam. Many orientations are brought into position for diffraction and a continuous trace of diffraction beam is directly obtained, which approximately correspond to a trace from a randomly oriented specimen.

Tsuruoka et al. have been applied both methods for an alloy containing two phases, austenite and ferrite (or martensite). ${ }^{3)}$ The method of Gullberg and Lagneborg is applied by calculating the average integrated intensity for austenite phase, $I \gamma$, from a set of $\{111\}$, $\{100\},\{110\},\{311\}$, and $\{331\}$ planes and that for ferrite (or martensite) phase, $I \alpha$ (or $I \alpha^{\prime}$ ), from a set of $\{110\},\{100\},\{211\},\{111\}$ and $\{321\}$ planes.

The agreement between X-ray diffraction and magnetic analysis was found to be very close, as shown in Fig. 1.

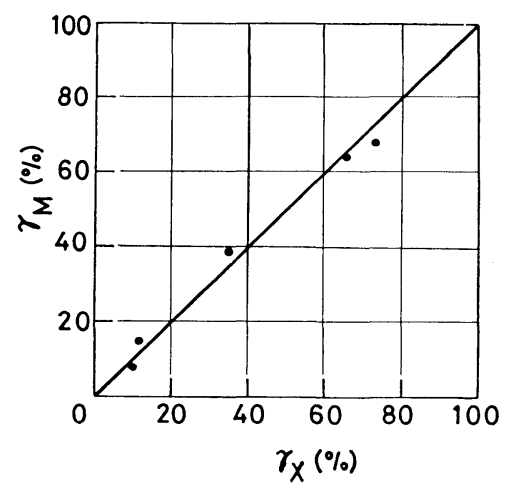

Fig. 1. Relationship between the volume fraction determined by the X-ray diffraction, $\gamma_{X}$, and that by the magnetic oscillation (the Vibrating Sample Magnetometer), $\gamma_{M}$.

\section{New Diffractometer for Application in Mill}

When the X-ray diffraction method is to be applied in the production lines, the following requirements must be satisfied for the instrument:

(1) Diffraction intensities are strong enough to minimize stastical counting errors.

(2) The measurement is to be carried out in a simple and reproducible way.

(3) Diffraction intensities are not substantially affected to the fluctuation of the distance between the instrument and a travelling material to be measured.

In general, it is very difficult to reduce the effect of the texture of a material in an on-line measurement.

Taking into account these requirements, a practical instrument was developed which could be applied in the production line; Figure 2 shows a schematic diagram of the principle of an angular dispersive X-ray diffractometer. Photograph 1 shows an inside view of a unit of X-ray source and detectors.

The X-ray diffractometer was equipped with three detectors. Detector 1 measures the diffraction inten-

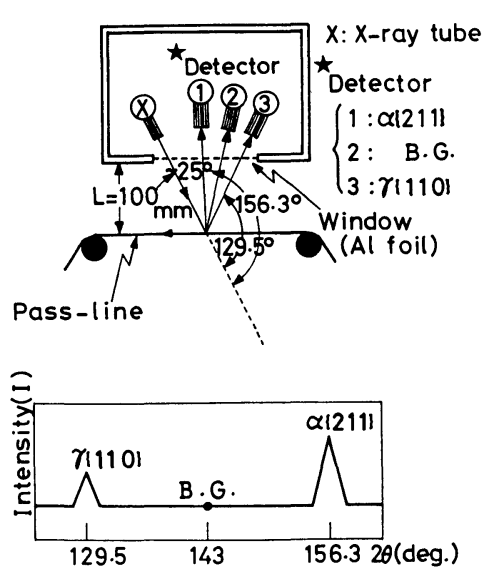

Fig. 2. X-ray diffractometer for on-line measurement. Schematic diagram of the X-ray technique using three detectors (Top), and schematic representation of diffraction patterns from a dual-phase steel, when a detector is scanned (Bottom).

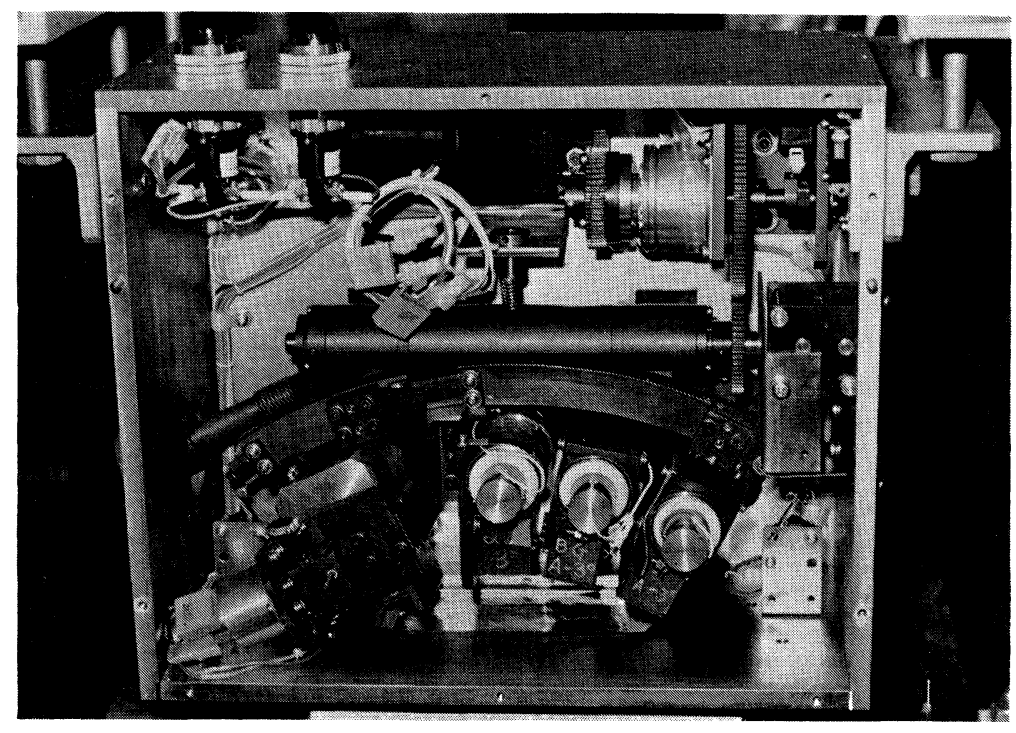

Photo. 1.

Inside view of the on-line diffractometer. 
sity from $\{211\}$ plane of martensite phase and detector 3 measures that from $\{110\}$ plane of austenite phase.

Detector 2 measures the background (BG) intensity. Detectors 1 to 3 are usually fixed at $2 \theta=156.3$ $\mathrm{deg}, 143.0 \mathrm{deg}$, and $129.5 \mathrm{deg}$, respectively. The position of detector 2 is precisely fixed while the position of detectors 1 and 3 can be manually adjustable within a few degrees in $2 \theta$. With detachment of detectors 1 and 2, detector 3 is ready to automatic scanning. " $X$ " in Fig. 2 denotes an X-ray source of chromium $\kappa \alpha$ radiation filtered with vanadium-foil. No Soller slit is attached in the path of incident beam. The divergent angles of a divergence and receiving slit are set at the same angle of $6 \mathrm{deg}$. Since the divergent angles of both slits are much larger than those used in a laboratory instrument, the intensity of incident beam is considerably high.

The dimensions of detector-windows are different to make the counting rate of detectors 1 to 3 at a ratio of $0.4,0.4$, and 1.0. The counting rate of detector 3 is set at largest because the intensity from metastable austenite phase is needed to be measured precisely in particular.

The divergence angles of the slits are so large that the diffraction intensities measured by this diffractometer are practically independent of a probable peak shift.

\section{Experiments}

The present experiments for application of quatitative phase analysis in the production line have been carried out in two steps: the first step to check performance of the new diffractometer in the laboratory by comparison with a conventional X-ray diffractometer and the second step to make the measurement in a mill.

\section{Performance Test in Laboratory}

Samples were prepared by sub-zero treatment to provide a variety of relative volume fractions of phases with a substantially identical texture.

The amounts of retained austenite, which were obtained by correcting the effect of textures by the method of Gullberg and Lagneborg, ${ }^{1)}$ are plotted against $I \gamma$ of $\{110\}$ austenite plane measured by the conventional diffractometer, in Fig. 3 and by the new diffractometer, in Fig. 4.

The results of both methods are very close.

For a given amount of retained austenite, the diffraction intensity was obtained about twenty times larger by the new diffractometer than by the conventional one, in spite of a lower operating power, a larger separation between the sample and the detectors, and a thicker filter of $K \beta$ radiation.

The new diffractometer was operated at a load of $4 \%$ of the maximum capacity, $3 \mathrm{~kW}$, of the X-ray tubes, while the conventional one at a load of $80 \%$. The unit of X-ray source and detectors in the new diffractometry shown in Photo. 1, was placed apart from the sample four times farther than in the conventional one.

\section{Application in Mill}

Tested materials were JIS type 301 stainless steel coils for the use of spring which had been rolled into several gages. X-ray analysis of retained austenite was carried out during uncoiling of the coils at a slitting line.

Photograph 2 shows the diffractometer set-up used in the present experiment.

The unit of X-ray source and detectors is suspended by a rigid frame, so that it can be rotated manually around a frame axis which is normal to the sheet surface. The unit can manually be set at an arbitrary position of the transverse direction of a strip. An aluminium foil with a thickness of $20 \mu \mathrm{m}$ was attached to the window to protect the diffractometer unit from a dusty atmosphere in the line. To cover the loss of $\mathrm{X}$-ray by attenuation in the aluminium foil, the power supply to the X-ray tube was increased to $25 \%$ of the maximum allowable load. Consequently diffraction intensities counted by the detectors were enough strong, amounting more than $10^{4} \mathrm{cps}$.

The travelling speed of the strip in this line is be-

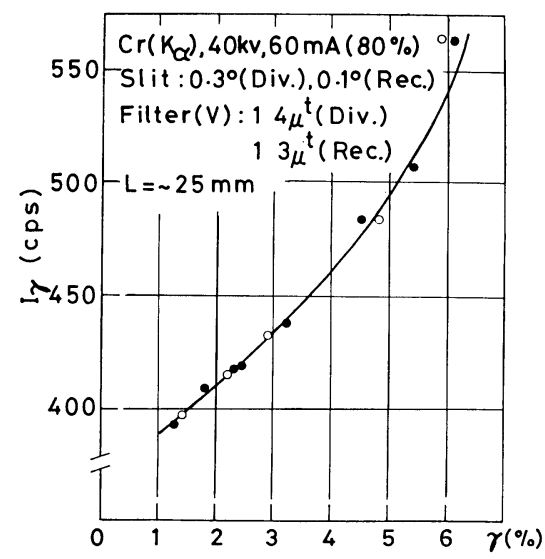

Fig. 3. Relationship between the volume fraction of retained austenite and the diffraction intensity from $\{111\}$ plane of austenite obtained by a conventional diffractometer.

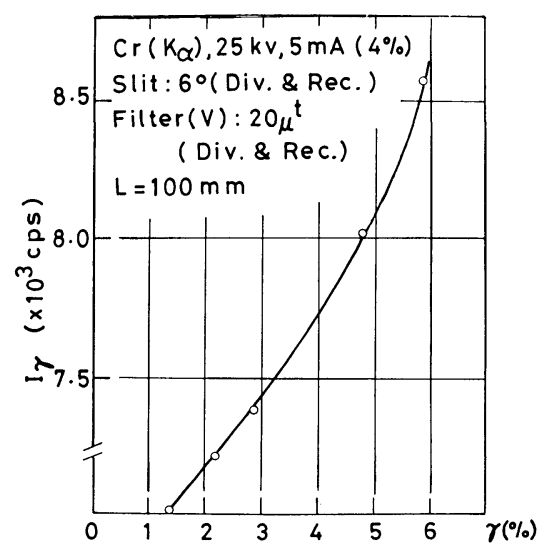

Fig. 4. Relationship between the volume fraction of retained austenite and the diffraction intensity from $\{110\}$ plane of austenite obtained by the on-line diffractometer (measured for the samples denoted by circle in Fig. 3 ). 


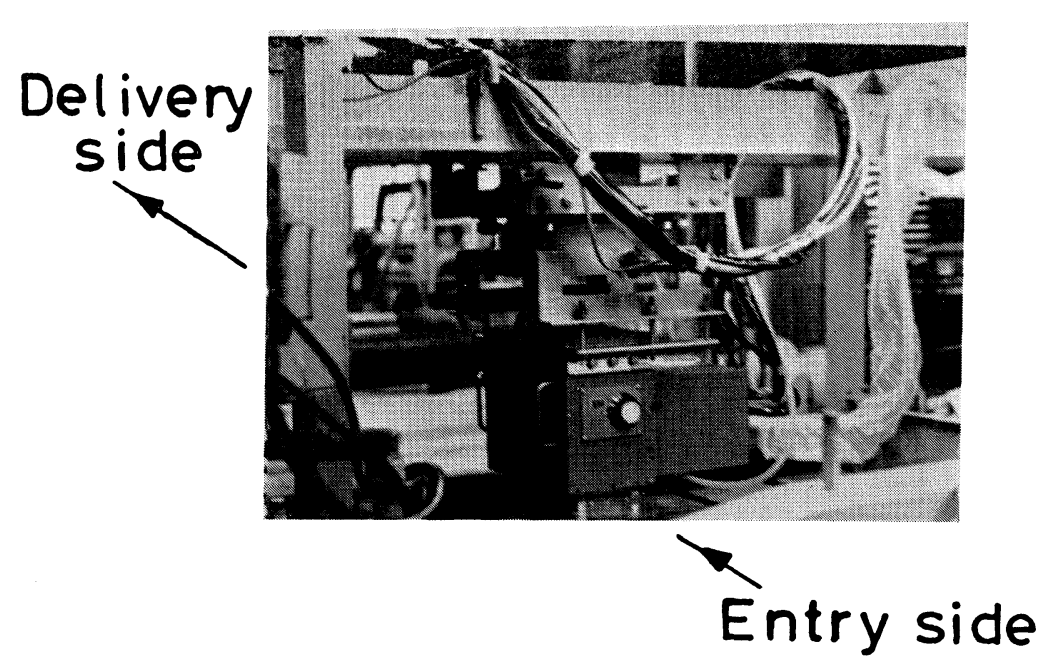

Photo. 2.

On-line diffractometer mounted in a production line. tween 20 and $40 \mathrm{mpm}$ (mpm: meters per minute). While the sample was travelling, the fluctuation of the distance between the detectors and the sample surface was within $\pm 1 \mathrm{~mm}$, so that the variation of diffraction intensity was within $\pm 0.5 \%$.

The details of the present experiments fall under following three items:

1) Confirmation of reproducibility of the measurement, by repeating five to ten times for each coil,

2) examination of the variation of volume fractions at various positions in the transverse direction of the strip, and

3) comparison of the diffraction geometry by arranging the equatorial plane of the strip, which is defined as a plane containing both the incident and the diffracting beams, parallel or vertical to the rolling direction.

At first, recording of diffraction intensity profiles from $\{110\}$ plane of austenite and $\{211\}$ plane of martensite on strip-chart was carried out with automatic scanning of detector 3, by interrupting the travel of strip at the starting and end point, and several positions at a certain interval in the intermediate part of a coil.

After fixing detectors 1 and 3 at the respective angular position of the peak intensity of $\{110\}$ diffraction from austenite and $\{211\}$ from martensite, continuous measurements of diffraction intensities were made together the background intensity measurements by detector 2 during the travel of strip.

Diffraction intensities received by each detector were recorded on strip-charts as counting rates (cps: counts per second) in the ratemeter mode and also read out manually from the registers of digital counters as integral counts in the fixed time mode.

\section{Results of Measurement in Mill}

X-ray diffraction intensities from austenite, $I \gamma$, were stationary along the length of coil of a given gage, except for small portions ranging about $10 \%$ of the total length from both top and tail ends of strip where intensities were weak.

The pattern of the distribution of diffraction inten-

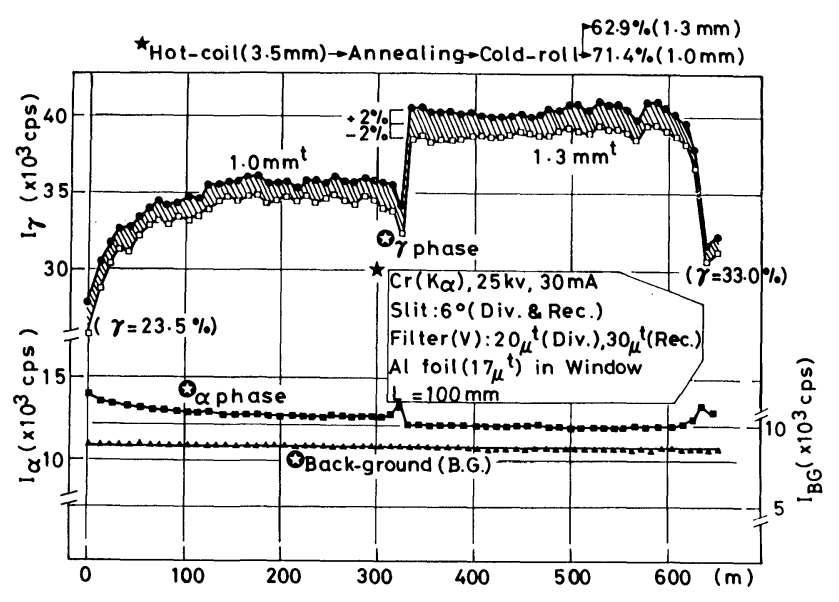

Fig. 5. A result of the on-line measurement, $I \gamma, I \alpha$ and $I_{B G}$ denote diffraction intensities from austenite and martensite and back ground intensity, respectively. Abscissa: Distance from the 'on-gage' starting point of the part of the thickness of $1.0 \mathrm{~mm}$.

sities from martensite phase, $I \alpha^{\prime}$, along the length of a coil was complementary to that of $I \gamma$.

Agreement between intensities obtained by the ratemeter and the fixed time mode were good, even though they were obtained in a different travelling pathes.

The reproducibility of the measurement was very satisfactory, even in both end parts of a coil where diffraction intensities changed abruptly as mentioned above.

Figure 5 shows a result of the measurements of a coil, cold rolled into two different gages with reductions of $62.9 \%$ for a half length of the coil and $71.4 \%$ for the other half from a hot rolled and annealed strip.

The measurement was carried out within the ongage parts of the coil along the center line of the width for ten times, and in this figure, the averaged values were plotted. The fluctuation range of $I \gamma$ was not greater than $\pm 2 \%$, including for both end parts, as shown by the hatched area of the figure. It was almost the same for martensite phase, while BG intensities remained stationary.

When the positions of measurement were changed 
in the width of the coil, diffraction intensities and their distribution patterns along the rolling direction of the coil coincided well with each other, including for both end parts of the coil.

The diffraction intensities from each phase, however, differ in accordance with the measurement geometry as shown in Fig. 6, which may be attributable to the presence of strong preferred orientations. The modes of diffraction geometry were to set the equatorial plane of the diffractometer parallel (mode (I)) or vertical (mode (II)) to the rolling direction.

The result shown in this figure was obtained at the center in the width of the same coil used in the previous test illustrated in Fig. 5.

At any position in the rolling direction, the diffraction intensity from austenite phase measured was weaker by mode (II) than mode (I). On the contrary, the diffraction intensity from martensite phase was obtained stronger by the measurement mode (II) than mode (I). In measurement modes (I) and (II), the distribution patterns of diffraction intensities from both phases, austenite and martensite, in the rolling direction of the coil were similar with each other, including for both end parts of the coil.

The background intensity $(B G)$, however, remained at the same level at any position, without regards to the measurement mode. From the fact that $B G$ intensity was stationary in the whole scope of measurements, the present measurements were proved to be performed in a very stable way.

In the on-line measurement, it is most important that the fluctuation of the distance between the diffractometer unit and the sample is kept as small as possible while the material is travelling, since diffraction intensities are directly influenced by change in the distance.

Therefore variation of diffraction intensities due to fluctuation of the distance was estimated by changing

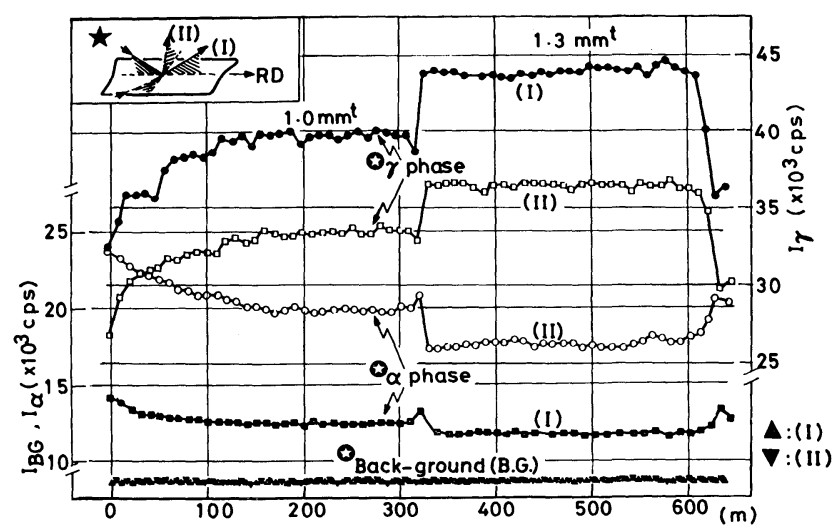

(I): the result parallel to the rolling direction

(II): parallel to the transverse direction

Fig. 6. Comparison between measurement modes. the clearance between pinch-rolls which were placed just before and behind the diffractometer unit.

When the fluctuation was within $\pm 3 \mathrm{~mm}$ for an average distance of $100 \mathrm{~mm}$ between the diffractometer unit and the sample, the variation of diffraction intensities was within $\pm 4 \%$.

\section{Discussion}

\section{Volume Fractions of Phases and Rolling Temperature}

The relation between the amount of strain-induced martensite and the rolling temperature will be discussed in terms of a factor ' nickel equivalent, ${ }^{34)}$ which indicates stability of austenite with regard to the chemical composition of the material.

It has been reported that the characteristics of metastable austenite phase is mainly determined by the nickel equivalent and, for a material having the nickel equivalent in a certain range of $19.5 \sim 20.9 \%$, the amount of strain-induced martensite strongly depends on the working temperature. ${ }^{4-7)}$

Table 1 shows the chemical composition of the material tested. The nickel equivalent of the material was calculated to be $20.0 \%$ from the equation proposed by Hirayama et al.,4) falling within the range in which the amount of strain-induced martensite changes by the working temperature. When the temperature of steel is not intentionally controlled to an appropriate level in the working process especially of a reversing mill, the temperatures of both end parts of a coil are lower than that of the middle part. Then the present findings that the ratio of the amount of martensite to that of austenite becomes smaller in the middle part of a coil, are a natural consequence of inhomogeneity of the rolling temperature of strip. Thus feasibility is suggested to measure relative amounts of phases and their variations along the whole length of coils in the production line.

\section{Effect of Texture}

It is well known that a cold-rolled material has some strong preferred orientations. As the X-ray diffraction intensity is directly influenced by the presence of preferred orientations, the effect of textures should be corrected to estimate more precisely the volume fractions of the phases.

In the on-line measurement, however, it is very difficult to correct the effect of textures, as mentioned before.

The present method which only compares the diffraction intensities from planes of a specific crystallographic type of each phases, austenite and martensite, in principle, disregards the influence of preferred orientation.

When the chemical composition, the reduction or temperature of rolling are controlled, the texture of

Table 1. Chemical composition of the material.

\begin{tabular}{ccccccccccc}
\hline $\mathrm{G}$ & $\mathrm{Si}$ & $\mathrm{Mn}$ & $\mathrm{P}$ & $\mathrm{S}$ & $\mathrm{Cu}$ & $\mathrm{Ni}$ & $\mathrm{Cr}$ & $\mathrm{Mo}$ & $\mathrm{Ti}$ \\
\hline 0.10 & 0.80 & 1.00 & 0.03 & 0.003 & 0.12 & 7.20 & 17.3 & 0.13 & 0.03 \\
\hline
\end{tabular}


the coil is regarded homogeneous through the whole length of a coil. In this case, fluctuations of the volume fractions of phases in the coil could be measured qualitatively by this method. By correcting the effect of the textures of the material, the volume fractions could be determined quantitatively.

In the present experiment, the rolling temperature was not stationarily controlled as mentioned in Section $V .1$, so that the texture is not homogeneous within a coil.

As the tested materials were commercial products, sampling was only made at both end parts of coils for measurements of textures in the laboratory.

The values, $\gamma=23.5 \%$ and $\gamma=33.0 \%$, shown in Fig. 5 were the volume fractions of retained austenite in both end parts obtained by correcting the effect of the textures.

Then the effect of working temperature on the rolling textures will be discussed with reference to the previous reports. Goodman and $\mathrm{Hu}$ reported the relations between textural component, crystallographic axis density parallel to the sample normal, and working temperature in a metastable austenitic steel. ${ }^{8,9)}$

The effects of cold rolling temperature on the texture and mechanical properties of a metastable austenitic stainless steel strip were reported by Oka and Kinoshita. ${ }^{10)}$ Figure 7 shows the relation between the textural component and the rolling temperature of the austenite phase, which agrees well with the result reported by Goodman and $\mathrm{Hu}^{8,9)}$ The $\langle 110\rangle$ axis density is about $20 \%$ lower after rolling at $200^{\circ} \mathrm{C}$ than at room temperature $\left(25^{\circ} \mathrm{C}\right)$.

Detector 3 measures the diffraction intensity from $\{110\}$ plane of austenite phase which is approximately parallel to the sample surface, as shown in the top of Fig. 2, providing the density in $\langle 110\rangle$ direction which is approximately normal to the sample surface.

In order to reduce the effect of texture, the random intensity, $I \gamma_{h^{\prime} k^{\prime} l^{\prime}}$, which is averaged to correct the diffraction intensity of $\left\{h^{\prime} k^{\prime} l^{\prime}\right\}$ plane for a material free of texture, must be obtained as described in Section $I I$.

$I \gamma_{110}$ can be calculated by dividing the diffraction intensity received in detector 3 by the $\langle 110\rangle$ axis density normal to the sample surface.

As shown in Fig. 7, $\langle 110\rangle$ axis density of austenite phase normal to the sheet surface of a cold rolled metastable austenitic stainless steel decreases, and the random intensity, $I \gamma_{110}$, increases with increasing rolling temperature.

If correction could be applied in the present case, the random intensities corrected from the intensities counted by detector 3 may be higher in the middle part than in both end parts of a coil.

Therefore the difference between the volume fraction of austenite phase in the middle and the end parts of a coil may increase.

However, without correction for textures as shown in Fig. 5, the present method is proved to be well worth applying to evaluation of material properties in production lines as a practical means. In particular it is an effective way to predict inhomogeneity of mechanical properties which is caused by the dif-

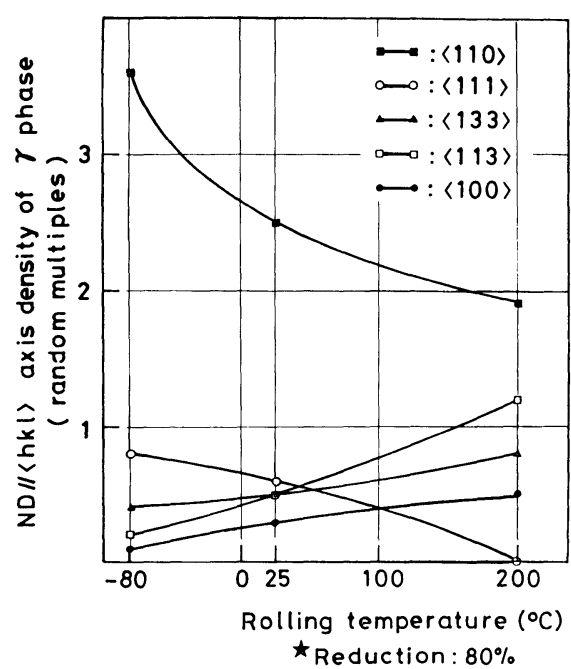

Fig. 7. Relationship between texture component and rolling temperature.

ference in rolling temperature.

\section{Conclusion}

Relative volume fractions of retained austenite and strain-induced martensite in a cold-rolled type 301 stainless steel were measured by an X-ray diffraction method in a slitting line of the steel coil.

A new unit of diffractometer was designed for application in the mill and verified for its performance in the laboratory with reference to the conventional diffractometer. Reproducibility of the present method was confirmed by repeated scanning of the whole coil length during uncoiling of the coil.

Diffraction intensities of austenite phase were weaker in the top and tail ends than in the middle part of the coil. This is well correlated with a conceptional distribution of retained austenite which can be deduced from the nickel equivalent and rolling temperature of the tested material, which suggests applicability of the present method in the production line, even though the effect of textures could not be taken into account.

The new X-ray diffractometer unit is proved to be successfully employed in the production mill as a practical means for prediction of inhomogeneity of material properties in the stainless steel strip for use of springs by monitoring the relative amount of austenite and martensite.

\section{REFERENCES}

1) R. Gullberg and R. Lagneborg: Trans. Met. Soc. AIME, 236 (1966), 1482.

2) R. L. Miller: Trans. ASM, 61 (1968), 592.

3) K. Tsuruoka, T. Abe and S. Katoh: Tetsu-to-Hagané, 56 (1970), S152.

4) T. Hirayama and M. Ogirima: J. Japan Inst. Metals, 34 (1970), 507.

5) T. Hirayama and M. Ogirima: J. Japan Inst. Metals, 34 (1970), 511.

6) T. Hirayama and M. Ogirima: J. Japan Inst. Metals, 34 (1970), 826. 
7) T. Hirayama and M. Ogirima: J. Japan Inst. Metals, 34 (1970), 892.

8) S. R. Goodman and H. Hu: Trans. Met. Soc. AIME, 230 (1964), 1413.

9) S. R. Goodman and H. Hu: Trans. Met. Soc. AIME, 233
(1965), 103.

10) Y. Oka and N. Kinoshita: Proceedings of the Sixth International Conference on Texture of Materials, ISIJ, Tokyo, (1981), 883. 